\title{
Alptraum oder Wirklichkeit?
}

\section{René Bloch}

Dr. med., Facharzt für Psychiatrie und Psychotherapie, Mitglied der FMH

Noch in der Mitte des 20. Jahrhunderts konnte man kaum behaupten, die Menschheit würde auf ihren Untergang zugehen in der Folge der negativen Nebenwirkungen des technischen Fortschritts. Nur eine kleine Zahl von Wissenschaftlern erkannte, dass die technische Entwicklung und die wirtschaftliche Expansion Gefahren für die Zukunft der menschlichen Gesellschaft schufen. Die wirtschaftlichen Vorteile der technischen Entwicklung und der wirtschaftlichen Expansion waren so eindeutig, dass man die Tatsache übersehen

\section{Warnende Stimmen wurden nicht gehört und gingen unter im Rausch des wirtschaftlichen Wachstums.}

Hinweis

${ }^{*}$ Der Autor dieses Beitrags hat in zahlreichen Beiträgen auf die Dringlichkeit von Massnahmen gegen den Klimawandel hingewiesen und zum Handeln aufgerufen. Neue Perspektiven zum Thema der Gefährdung der Schöpfung und der Stellung des Menschen hat er in seinem

Buch «Destruktionstrieb und Transzendenz», 2017, veröffentlicht.

* Bei Drucklegung dieses Textes wurde von der World Meterological Organisation WMO bekanntgegeben, dass die Konzentration der Treibhausgase in der Atmosphäre einen neuen Höchststand erreicht hat. Die WMO warnt vor katastrophalen und unumkehrbaren Folgen Siehe dazu: https:// public.wmo.int/en/ media/press-release/ greenhouse-gas-levelsatmosphere-reach-newrecord

Jährlicher Klimabericht der WMO: https:// public.wmo.int/en/ourmandate/climate/wmostatement-state-ofglobal-climate hat, dass einmal die Grenzen des Wachstums erreicht sein würden und die Ressourcen und energetischen Reserven des Planeten nicht unendlich wären. In den Nachkriegsjahren befand man sich in einer Euphorie des Wiederaufbaus, und in den folgenden Jahrzehnten wurde versucht, den ganzen Planeten am neuen Reichtum durch Globalisierung teilhaben zu lassen. Warnende Stimmen wurden nicht gehört und gingen unter im Rausch des wirtschaftlichen Wachstums.

Heute meint man, schlecht zu träumen, wenn in den Medien davor gewarnt wird, dass das Ökosystem des Planeten mit allen Folgen für die Existenzgrundlagen der Menschheit zusammenbrechen könnte. In der Folge der Ausbeutung des Planeten und dessen Verschmutzung und Erwärmung sind die natürlichen Gleichgewichte schon stark in Mitleidenschaft gezogen worden, und die Möglichkeit eines Kollapses ist in die Nähe gerückt.

Seit circa zehn Jahren wird man sich der Gefahren bewusst, und weltweit sind bereits umfangreiche Massnahmen ergriffen worden, um eine weitere Schädigung des Lebensraumes und katastrophale Auswirkungen zu vermeiden. Die Massnahmen, die ergriffen werden müssen, benötigen ein Zusammenwirken aller Mitglieder der internationalen Völkergemeinschaft. Ein schwieriges Ziel, dessen Erreichung kaum vorstellbar ist. Erst kürzlich hat der Weltklimarat zur Einigkeit aufgerufen, um das Ziel einer maximalen Erderwärmung von 1,5 Grad zu erreichen. Der Augenblick sei gekommen, da sofortiges und entschlossenes Handeln gefordert sei und nicht nur die Erklärung guter Absichten.
Die Warnungen des Klimarats sind angesichts der bereits eingetretenen negativen Klimaveränderungen unüberhörbar. Umso erstaunlicher sind die politischen Reaktionen, die sich zwar für die geforderten Klimaziele einsetzen wollen, gleichzeitig aber zahlreiche Bedenken gegen sofortige drastische Massnahmen ins Feld führen. Die Freiheit des Handelns wird eingeschränkt durch die Furcht vor schwindendem Wirtschaftswachstum und Arbeitslosigkeit. Die Anforderungen, die die heutige Situation stellt, sind schwer realisierbar ohne bedeutende soziale Umwälzungen. Allerdings ist diese Krisensituation dem Umstand zuzuschreiben, dass man zu lange zugewartet hat, um etwas gegen die Fehlentwicklung der Beziehung des Menschen zur Natur zu unternehmen. Wenn es heute nicht gelingt, die Prioritäten richtig einzuschätzen und der Rettung der Natur und der ökologischen Gleichgewichte einen absoluten Vorrang zu geben, könnte es durchaus sein, dass es morgen zu spät ist und es zu einem Kollaps der Ökosysteme mit einem Untergang des meisten Lebens kommt. Die Menschheit steht heute in ihrer Gesamtheit vor einer der schwierigsten Entscheidungen ihrer Geschichte: Entweder werden weltweit sofort Massnahmen gegen die weitere Zerstörung der natürlichen Umwelt ergriffen und man kann eine chaotische Entwicklung vielleicht noch aufhalten, oder man versucht, den erreichten Wohlstand für einige Jahrzehnte zu erhalten ohne Rücksicht auf die Folgen für die Zukunft.

Die Menschheit steht heute in ihrer Gesamtheit vor einer der schwierigsten Entscheidungen ihrer Geschichte.

Parallel zum Kampf gegen den anthropogenen Klimawandel müssen Massnahmen zur Beherrschung von dessen gesundheitlichen Auswirkungen unternommen werden. Der Klimawandel hat ausgedehnte Folgen für die Gesundheit einzelner Menschen und für die gesamte Bevölkerung. Unter dem Einfluss thermischer Belastungen oder von Luftschadstoffen hat die Mortalität mehrerer Krankheiten zugenommen. Die Erforschung der gesundheitlichen Auswirkungen des Klimawandels ist relativ weit fortgeschritten im Vergleich mit der Erforschung der möglichen Massnahmen gegen denselben, da solche zum Teil schwer realisierbar und tabuisiert erscheinen. 